\title{
RELACION ENTRE LA ABUNDANCIA RELATIVA Y DENSIDAD REAL EN TRES POBLACIONES DE AVES.
}

Zulema Quinteros Carlos ${ }^{1}$, Edgar Sánchez Infantas ${ }^{1}$ y Humberto Tovar Serpa ${ }^{2}$

\section{Resumen}

Se evaluó la relación entre el Indice de Abundancia Relativa al Espacio (IARE) y la Densidad Real (DR) - estimada mediante las series de Fourier - en tres poblaciones de aves de importancia económica - Forpus coelestis, Columbina cruziana y Mimus longicaudatus - de la Región Grau, Perú.

En el análisis de las relaciones existentes se empleó la Regresión Lineal Simple tomando como variable dependiente a la Abundancia Relativa y como variable independiente a DR. La significación de la Correlación se evaluó mediante su Coeficiente de Correlación. Los resultados sugieren que para estas condiciones, la Abundancia Relativa es un buen estimador de la Densidad Real. Sin embargo, se ha encontrado que ciertos modelos no lineales describen de mejor manera que un modelo lineal la relación IARE-DR, determinándose puntos (IARE o DR) a partir de los cuales se deberá tener especial cuidado para descartar la existencia de relaciones no lineales, tanto en el nivel extensivo como intensivo, en la medida que el uso del modelo lineal implicaría una sobre o subestimación de la densidad.

Palabras clave: Abundancia relativa, Densidad Real, Abundancia poblacional.

\begin{abstract}
In this research, we analysed the linear relationship between Space Relative Abundance Index (SRAI) and Real Density (RD) by means of Fourier series in three bird populations of economic importance: Forpus coelestis, Columbina cruziana and Mimus longicaudatus from the Region Grau, Peru.

In the analysis of the relationships, Lineal Regression was used, with the relative abundance as the dependent variable and RD as the independent variable. The significance of the correlation was assessed by the coeficient of correlation. Results suggest that, under prevalent conditions, Relative Abundance is a good estimator of the RD. However, some non linear models describe in a better way the relationship between SRAI and RD. Only certain points (SRAI or RD) should be taken into account when excluding non linear relationships, both at the extensive and intensive level, because the linear model could under estimate or over estimate the density.
\end{abstract}

\section{Introducción}

En el manejo de las poblaciones animales el punto inicial de cualquier programa suele ser la evaluación poblacional, ya que la variabilidad espacio temporal de las mismas es un tema complejo (McArdle et al., 1990; Pimm,1991 y Pimm \& Redfearn,1988).

Asimismo, Caughley (1977) señala, que el manejo de las poblaciones está asociado con la densidad de las mismas: alta, media y baja. Por lo tanto la alternativa es el control, la cosecha y la protección poblacional, respectivamente.

Para el caso de la avifauna terrestre peruana, la generación de beneficios económicos por la extracción comercial para la exportación de mascotas, y el efecto sobre las poblaciones silvestres han sido documentados (Falero y Sánchez, 1990; Rosales, 1999). Cosa similar se ha hecho para las poblaciones de aves plaga aún cuando se requieren mayores niveles de generalización (Quinteros, 1992).
En todos los casos mencionados es importante conocer no sólo el estado presente de la población sino también sus tasas y estilos de cambio, el mismo que puede ser consecuencia de procesos de regulación interna o de efectos de la variabilidad ambiental (Murdoch, 1994; Sinclair \& Peach, 1996), los que en nuestro medio están asociados a la presencia de eventos como El Niño (Sánchez y Quinteros, 1997; Torres y La Torre, 1994).

En este contexto, las estimaciones de la densidad real de una población resultan poco prácticas porque por sus costos y dificultades no se las puede aplicar de ordinario; sin embargo, los Indices de Abundancia Relativa se presentan como alternativa. Se plantea, por tanto, que más que una estimación muy exacta de la densidad de una población, se necesita una serie de estimaciones que mantengan su inexactitud constante y por tanto sean comparables entre sí tanto en el espacio como en el tiempo. Parte de mantener constante la inexactitud es garantizar que la relación

\footnotetext{
${ }^{1}$ Laboratorio de Ecología de Procesos, Departamento de Biología. Universidad Nacional Agraria La Molina. Av. La Universidad S/N. La Molina. Apartado 456 Lima zquinteros@lamolina.edu.pe,

${ }^{2}$ Especialidad en Conservación de Recursos Forestales, Escuela de Post-Grado. UNALM.
} 
que guarde el índice con la densidad real que pretende estimar, se mantenga para un conjunto muy amplio de densidades. Esto se conseguiría si la mencionada relación fuese lineal siempre (Sánchez et al., 1998).

La presente investigación tuvo por objetivo evaluar si la relación entre el Indice de Abundancia Relativa al Espacio (IARE) y la Densidad Real (DR) es lineal en poblaciones de aves de importancia económica de la Región Grau, tomando como ejemplos a Forpus coelestis (FORCO), Columbina cruziana (COCRU) y Mimus longicaudatus (MILO). Se evaluaron además las condiciones en las que se altera esta linealidad y las consecuencias derivadas de ello.

La evaluación, en el nivel extensivo, se llevó a cabo utilizando las carreteras o trochas secundarias de los ex - Distritos Forestales Tumbes y Sullana, en los departamentos de Tumbes y Piura, respectivamente de la Región Grau. El nivel intensivo se evaluó en un sector del "Coto de Caza El Angolo", Provincia de Sullana.

\section{Revisión bibliográfica.}

El Indice la Abundancia Relativa al Espacio (IARE) es una variable relacionada a la Densidad Real (DR) de una población y que por eso permite conocer cómo cambia la población aún cuando nunca pueda conocerse el tamaño real de esta. Es justamente la posibilidad de aplicarlos de modo extensivo lo que les da a estos índices una gran utilidad en la evaluación de poblaciones con una gran variabilidad espacio - temporal (Caughley, 1977; Norton Griffiths, 1978; Sánchez et al., 1988).

La relación más general puede expresarse así: Densidad $=\mathbf{a}+\mathbf{b}$ Índice. Esta expresión muestra una relación lineal y por tanto puede ser ajustada a partir de una serie de datos experimentales para conocer los valores de a y b. Sin embargo, la relación podría no ser lineal, en cuyo caso se necesitaría una transformación inicial de los datos.

Verificar la linealidad de la relación, nos asegura la validez del Índice para detectar cambios en la densidad real aún cuando estos sean de gran magnitud. De lo contrario la utilidad del Índice estaría restringida a detectar cambios de poca cuantía ya que incurriríamos en errores de estimación (Caughley, 1977).

De los múltiples métodos disponibles para la estimación de DR (Tellería, 1986) se ha escogido una versión particular de los conteos directos: los transectos en línea. La selección del método obedece a que los supuestos sobre los que se basa son de fácil cumplimiento cosa que no sucede con los otros métodos.

La metodología de los transectos en línea establece (Burnham et al., 1980) que se deben ubicar randomizadamente una serie de líneas en el espacio que se desea evaluar. Cada línea se recorre registrando los animales ubicados en ambos lados.

Los registros que se hacen por cada individuo visto son:

- Distancia perpendicular del individuo a la línea de recorrido (x).

- Distancia de observación o distancia entre el observador y el individuo (r).

- Ángulo de observación (é), que es el ángulo que forma la línea de recorrido con la línea entre el observador y el individuo registrado.

En la práctica no suelen tomarse los tres datos; se registra x ó r y é. De hecho, a partir de r y é se puede calcular $\mathrm{x}(\mathrm{x}=\mathrm{r}$ sen é). Como señalan Burnham et al. (1980), la validez del presente método descansa en cuatro supuestos:

1. Los individuos ubicados sobre la línea de recorrido nunca se dejarán de registrar, es decir, su probabilidad de ser vistos es uno (1).

2. Los individuos se registran en su posición inicial; esto implica que se los registra antes de que puedan desplazarse alejándose o acercándose a la línea. Adicionalmente, ningún individuo es contado dos veces.

3. Las distancias y los ángulos se miden exactamente, es decir sin error.

4. Las observaciones son eventos independientes.

El orden de los supuestos expresa su importancia relativa. La tarea principal es la determinación de la visibilidad de los animales en función de su distancia de la línea de recorrido. Se supone que esta probabilidad de detección disminuye mientras más lejos se encuentra un individuo de la línea. Basado en esta función se determina el ancho efectivo de la faja evaluada, a partir de la cual se estima la densidad real.

\section{Metodología \\ Determinación de IARE}

En la Evaluación Extensiva, las Unidades Muestrales (UM) fueron de longitud variable con inicio y final definido por la presencia de un pueblo que figuraba en el Mapa Departamental. La evaluación consistió en recorrer la UM, con una camioneta con un anotador y dos observadores, registrando los individuos que se avistaban en una faja de ancho referencial, de 50 metros a ambos lados de la carretera. Las unidades en que se expresan los IARE corresponden a "individuos por kilómetro" lineal de recorrido. Por tanto, no se hace mención al área sino a la longitud recorrida, lo que explica porqué el ancho de la faja de observación es meramente referencial.

Dado que los IARE, son índices operacionales (definidos por el método mismo de medida), es particularmente importante que los métodos de medida se mantengan constantes. Esto es, que el sesgo de estos índices se mantenga constante. En la práctica esto significó mantener constantes: la velocidad de recorrido de la camioneta (40 kph), horarios de 
evaluación (periodo matinal de 07:30 - 11:00) y un mismo grupo de observadores.

En la Evaluación Intensiva, las UM fueron transectos cuyo inicio y fin se establecieron con referencia a puntos geográficos. La longitud total de cada uno de estos transectos se estableció en tres kilómetros y medio (medidos en tramos de 100 metros). Al interior de cada uno de los transectos se construyeron las UM agrupando tramos de 100 metros hasta conseguir un número de al menos 20 individuos sin importar la longitud de la UM. Se procedió de esta manera para satisfacer una exigencia de los estimadores de Fourier.

Las observaciones se hicieron recorriendo a pie los transectos, registrando el número de aves que se veían a ambos lados de la línea, manteniendo de modo referencial un ancho aproximado de 30 metros. Las unidades fueron "individuos por 100 metros" de recorrido. La identificación y ubicación de los grupos de aves se hizo normalmente a ojo desnudo, recurriendo al uso de binoculares sólo cuando se requería confirmar esta información. Para garantizar el mantenimiento de un sesgo constante se mantuvieron constantes las siguientes condiciones de operación: los conteos se realizaron siempre entre las 08 y 10 de la mañana, a una velocidad aproximada de entre 3 y 5 minutos por cada 100 metros y manteniendo el mismo grupo de observadores.

\section{Determinación de DR.}

Usando de base las mismas UM que se emplearon para la determinación de los IARE - y de modo simultáneo -, se registró para cada una de ellas la información necesaria para estimar DR en transectos con ancho variable (Burhann et al., 1980; Krebs, 1989).

Esta técnica se basa en la construcción de una función de detección de los animales ( $\mathrm{f}(0)$ ), que varía con la distancia de estos al eje del transecto, suponiendo que la probabilidad de detección de un animal que se encuentre sobre el eje mismo será uno (1) y que irá disminuyendo conforme la distancia se incremente. Por lo tanto, se registraron las aves y sus distancias a la línea de recorrido. En el caso de grupos de aves se tomó como referencia para medir la distancia del grupo a la línea, aquella que había entre el punto central del grupo y la línea de recorrido.

Las estimaciones de las distancias en todos los casos se hicieron a simple vista pero luego de un entrenamiento previo. Como indican Burnham et al. (1980) es conveniente establecer una distancia máxima o distancia de truncamiento que para la evaluación extensiva se fijó en 50 metros y para la evaluación intensiva en 30 metros, distancias que se fijaron en función de la visibilidad.

La función de detección $\mathrm{f}(0)$ en el método de Fourier, permite calcular el ancho efectivo de la faja y por lo tanto estimar la Densidad Real. El modelo general para estimar la densidad tiene la siguiente forma: $\mathbf{D}=\mathbf{n} / \mathbf{2} \mathbf{L a}$, donde $\mathrm{D}=$ densidad, $\mathrm{L}=$ longitud del transecto, $\mathrm{a}=$ ancho real estimado y $\mathrm{n}=$ número de individuos registrado en el transecto. Si se pone a la densidad con base a la función de detección esta fórmula toma la siguiente forma: $\mathbf{D}=\mathbf{n} \mathbf{f}(\mathbf{0}) / \mathbf{2 L}$, siendo $\mathrm{f}(0)$ la probabilidad de detección cuando el individuo se encuentra sobre el eje del transecto.

Cuando se emplea esta metodología para estimar densidades, las UM se distribuyen siguiendo algún criterio de randomización lo que permite que previo cálculo de la variancia de D, se construyan Intervalos de Confianza para las estimaciones de la densidad media o el número total de individuos del área en estudio. En el presente caso sin embargo no se trata de estimar la densidad en ninguna área, sino sólo de conocer el valor que ésta toma en cada UM. Esto es así porque el interés está en analizar la relación de DR con la que corresponde a la Abundancia Relativa, siendo los elementos de comparación las UM, con lo que los cálculos de variancia y las estimaciones por intervalos no son necesarios.

Las estimaciones de $f(0)$ empleando series de Fourier y ak se hacen con las siguientes expresiones:

$$
\begin{gathered}
\mathbf{f}(\mathbf{0})=\underset{\mathbf{w}^{*}}{\mathbf{1}}+\sum_{\mathbf{k}=1}^{\mathbf{m}} \mathbf{a}_{\mathbf{k}} \\
a_{k}=\left[\frac{2}{n w^{*}} \sum_{i=1}^{n} \cos \left(\frac{k \pi}{w^{*}}\right)\right]
\end{gathered}
$$

donde :

$\mathrm{k}$ es el número correspondiente al término de la serie de Fourier y $\mathrm{x}_{\mathrm{i}}$ es la distancia del individuo i a la línea de transecto.

El cálculo de los valores correspondientes a $a_{k}$ se hizo en una hoja de Excel. Según recomiendan Burnham et al. (1980) el número de términos de la serie de Fourier a emplear en cada estimación no debe ser mayor de 6. Indican que mientras más términos se emplee mejorará la calidad del estimador pero se incrementa su varianza. Los mencionados autores sugieren el empleo de una regla de detenimiento (stoping rule) que marca el número de términos a usar. Esta regla establece que:

$$
\stackrel{1}{\mathrm{~W}^{*}}(2 / \mathrm{n}+1)^{1 / 2} \geq\left|\mathrm{a}_{\mathrm{m}+1}\right|
$$

Las densidades se han determinado en individuos por hectárea. No se han computado variancias para estos estimados, puesto que no se los ha empleado para estimar la densidad total de la zona de estudio y por lo tanto no se requería contar con intervalos de confianza.

Determinación del tipo de relación entre IARE y DR. Con el fin de analizar la linealidad IARE-DR para un conjunto de situaciones lo más amplio posible, las 
unidades muestrales (UM) se han ubicado buscando copar zonas de alta y baja abundancia, las que se han determinado con base en registros y mapeos previos (Sánchez et al., 1992). Por ejemplo, en la evaluación extensiva de FORCO, la UM de más alta abundancia supera en más de 60 veces a la UM de menor abundancia, cubriendo así un espectro suficientemente amplio de abundancias. En MILO esta relación es de 35 a 1.

El análisis empleado es la regresión lineal simple tomando como variable dependiente a IARE y como variable independiente a DR, escogida de acuerdo al Modelo I. La significación de la correlación se evaluó mediante su Coeficiente de Correlación contrastándolo con un Cuadro de significación (Steel y Torrie, 1985). En cada caso se elaboró el correspondiente Diagrama de Dispersión.

Adicionalmente, se buscaron relaciones no lineales de tipo potencial, logarítmico y exponencial, comparándose los resultados obtenidos de los ajustes con aquellos correspondientes al Modelo Lineal Simple. El criterio de comparación fue siempre el coeficiente de correlación respectivo. En esto se siguieron las recomendaciones de Daniel y Wood (1980).

Estimación del sesgo debido a relaciones no lineales.

Los IARE como estimadores de DR tienen un sesgo que se mantiene constante si la relación entre ambas variables se mantiene constante para todo el espectro de densidad real bajo interés. En ese sentido, alejamientos del Modelo Lineal habrán de generar problemas de sobre y subestimación, que dependerán de la naturaleza misma de la no linealidad y de los parámetros que la definen.

En el presente caso se han analizado estos efectos tomando como base la línea recta obtenida por regresión, contrastando contra ella los resultados de ajustar los mismos datos a diferentes relaciones no lineales (exponencial, logarítmica y potencial), dando preferencia a los modelos que mejor ajuste daban. La diferencia entre los valores predichos por la ecuación lineal y aquellos que fueron simulados de acuerdo a cada modelo no lineal, es un estimador de la magnitud de la sobre o subvaluación en que incurre respectivamente cada modelo. Se ha establecido, además, un límite para la sobre o subestimación teniendo presente que de ordinario los intervalos de confianza construidos para el IARE deben ser menores o iguales al $10 \%$ de éste. Esta es una regla a emplear en todas las operaciones de muestreo de poblaciones (Norton - Griffiths, 1978).

Para los valores del IARE estimado según el modelo lineal se construyeron intervalos de confianza (superior e inferior) que representan $\pm 10 \%$ del valor correspondiente del IARE. Dado que esta es una variación de magnitud aceptada por los estándares ya citados, se estableció el criterio por el cual, si el valor predicho por un modelo no lineal del IARE se encuentra dentro de este intervalo de confianza del IARE lineal, se asume que no hay diferencias entre el modelo lineal y el no lineal y por tanto se usa el primero. Esta operación permitió además establecer un rango de densidades para el cual el alejamiento del modelo lineal no causa graves problemas de sobre o subvaluación.

\section{Resultados y discusión. \\ IARE de las especies registradas.}

En la Tabla 1 se presenta la abundancia relativa (individuos por kilómetro de recorrido) para las dos especies de aves (FORCO y MILO) estudiadas en la Evaluación Extensiva y en la Tabla 2 para las dos especies de aves (FORCO y COCRU) estudiadas en la Evaluación Intensiva.

La información de abundancia obtenida para FORCO en la evaluación extensiva, es similar a la que obtuvieron Sánchez et al. en 1988 para el tramo Sauce Grande - El Angolo, Encuentros - Bejucal y Bejucal Jahuay Negro. De manera general, los resultados muestran pocos tramos con mucha abundancia y muchos tramos con poca abundancia. Este mismo comportamiento se presenta para MILO y las dos especies - FORCO y COCRU evaluadas en el nivel intensivo.

\section{Densidad Real de las especies registradas.}

La matriz empleada para el cálculo de la densidad real (DR) empleando las series de Fourier, construida según las sugerencias de Burnham et al. (1980) se muestra en la Tabla 3 tomando de ejemplo al tramo Sauce Grande - El Angolo.

La primera columna contiene el número de orden de cada uno de los individuos registrados; la segunda columna $\left(\mathbf{x}_{\mathbf{i}}\right)$ contiene sus correspondientes distancias. Las siguientes columnas contienen los términos de la serie de Fourier empleados posteriormente para la estimación de DR. El término $\mathbf{L}$ hace referencia a la longitud en metros de la unidad muestral y el término W se refiere a la distancia de truncamiento, es decir la distancia máxima de observación, la misma que para la evaluación extensiva se fijó en 50 metros y para la evaluación intensiva en 30 metros.

Las columnas signadas con $\mathrm{k}=1, \ldots, \mathrm{k}=6$, representan los términos de la serie de Fourier, cuyas sumas se tienen en la fila de totales. No todos los términos se usan en la estimación, siendo el término sr el que hace referencia a la regla de detención, la misma que fija el número de términos de la serie que se emplean para la estimación de la DR. Finalmente se presenta la información sobre densidad en metros cuadrados (D) y en hectáreas (DR).

El empleo de los transectos en línea para la determinación de DR supone que la probabilidad de detectar un individuo es más alta cuanto más cerca está a la línea de recorrido, siendo esta probabilidad uno cuando el individuo está sobre la línea de recorrido. Una evaluación gráfica de este supuesto se 
realizó construyendo histogramas que para un intervalo de clase de cinco metros, mostraron cómo se distribuyeron los individuos en función de su distancia a la línea de recorrido. De manera general, los histogramas mostraron que la mayor cantidad de individuos se encuentra cerca a la línea de recorrido. De hecho, en la mayoría de los casos alrededor del $80 \%$ de los individuos se encuentran dentro de los 25 primeros metros (recuérdese que la distancia de truncamiento es de 50 metros en la evaluación extensiva y 30 metros en la intensiva).

En las Tablas 1 y 2 se presenta los datos estimados de densidad real para las especies de aves estudiadas en la Evaluación Extensiva e Intensiva.

\section{Relación entre DR - IARE.}

Como se indicó, la estrategia que se usó para evaluar la relación entre DR - IARE fue la Regresión Lineal, teniendo como variable independiente a DR y como variable dependiente a IARE. Por tanto, los resultados obtenidos en esta sección tienen la forma de Coeficientes de Determinación $\left(\mathrm{R}^{2}\right)$ y Coeficientes de Correlación (r) para el Modelo Lineal y para Modelos No Lineales de tipo Potencial, Exponencial y Logarítmico.

En la evaluación extensiva los resultados de los coeficientes de determinación y correlación de FORCO mostraron que todos los modelos, excepto el exponencial, alcanzan significación al 5\%, el valor más alto para el coeficiente de correlación se tiene en el modelo lineal (0.8027). El diagrama de dispersión correspondiente reforzó la sugerencia, que el modelo lineal es una buena descripción para la relación DR IARE (Tabla 4, Figura 1).

De este modo, se verifica la existencia de una relación lineal entre DR y su correspondiente IARE. Sin embargo, conviene tener presente que existe al menos dos modelos no lineales que también explican los mismos datos con significación estadística, aunque con menores coeficientes de correlación. Por lo tanto, el argumento por el cual se debería preferir el modelo lineal frente a los no lineales es el que corresponde al principio de la parsimonia (Navaja de Ockham) (Hutchinson, 1981), es decir el principio que plantea que si dos modelos de complejidad diferente explican un mismo conjunto de datos, debemos escoger el de menor complejidad.

En el caso de MILO (Tabla 4), el modelo lineal, también, alcanza significación estadística al 5\%. Dos modelos no lineales (potencial y exponencial) alcanzan un mayor nivel de significación estadística (1\%), por lo que explicarían mejor la relación. Si se examina el diagrama de dispersión (Figura 1), sin embargo, éste sugiere que el modelo lineal describe muy bien la relación DR - IARE.

Por lo demás, los mensajes que dan los modelos exponencial y potencial son exactamente opuestos: una curva cóncava en el primer caso y una curva convexa en el segundo (Figura 1), acentuándose las diferencias cuanto más altas son las densidades. En ese sentido el modelo lineal representa una especie de promedio entre ambos extremos, razón por la cual es razonable asumirlo como el que mejor representa la relación entre las variables estudiadas.

Por lo tanto, se puede decir que el modelo lineal es el que mejor describe la relación entre DR e IARE para FORCO y para MILO, pudiéndose por tanto emplear los IARE como estimadores de DR para evaluaciones extensivas de estas especies y especies similares.

En el caso de la evaluación intensiva, en la Tabla 5 se consignan las valores de los coeficientes de determinación y correlación para los modelos lineal, logarítmico, potencial y exponencial indicándose su significación estadística, para ambas especies.

Resulta notable que todos los modelos empleados en el caso de FORCO tengan alta significación estadística $(\mathrm{p}<0,01)$ lo que en principio haría de todos ellos buenos candidatos para explicar estos datos. Sin embargo, en parte por el criterio del principio de la parsimonia y en parte por que el coeficiente de correlación del modelo lineal es algo mayor, parece razonable escoger a este modelo como el mejor descriptor de la relación entre DR e IARE.

Los resultados de COCRU indican que a excepción del modelo logarítmico todos los demás alcanzan significación estadística $(\mathrm{p}<0,05)$. El modelo lineal es el que tiene el segundo valor más alto para su coeficiente de correlación $(\mathrm{r}=0,6113)$, estando por detrás del modelo exponencial $(\mathrm{r}=$ 0,6288 ). Nuevamente sobre la base del principio de la parsimonia y el diagrama de dispersión estamos en posición de escoger el modelo lineal como un descriptor apropiado de la relación DR - IARE.

Estimación del sesgo debido a relaciones no lineales.

En el nivel extensivo, para FORCO el modelo logarítmico es el modelo no lineal que mejor correlación alcanza $(\mathrm{r}=0,7908, \mathrm{p}<0,05)$ al compararse con el modelo lineal $(\mathrm{r}=0,8027, \mathrm{p}<0,05)$. Adicionalmente el modelo potencial alcanza un valor alto para su correspondiente correlación $(\mathrm{r}=0,7246$, $\mathrm{p}<0,05$ ). Al mismo tiempo, para cada uno de estos casos se ha construido un gráfico en el que se comparan los valores esperados para un IARE lineal (con sus intervalos de confianza de $\pm 10 \%$ ), con los que surgen del empleo de un IARE no lineal. La Figura 3 muestra esta comparación para el modelo logarítmico y la Figura 4 lo hace para el modelo potencial.

De acuerdo a la Figura 3, si la relación DR - IARE fuese logarítmica, se incurriría en errores de sobre estimación para IAREs inferiores a un valor de alrededor de 1,8 ind/Km. Esto implica que el IARE lineal está reportando valores que están por encima de los que corresponden al IARE logarítmico. Para IAREs entre 1,8 y $3,9 \mathrm{ind} / \mathrm{Km}$ aproximadamente, el 
problema es más bien de subestimación. Por encima de 3,9 ind $/ \mathrm{Km}$ el problema sería nuevamente una sobreestimación. Estos valores estarían marcando los puntos críticos que señalan cambios en el comportamiento de los estimadores.

Strictu sensu la Figura 3 está indicando que no existen rangos en los cuales el IARE lineal sea válido, pues pasa de problemas de sobre a subestimación alternativamente. Sin embargo, debe tenerse en cuenta que anteriormente se había aceptado que el modelo lineal describía bien la relación DR - IARE para un rango de densidades entre 0 y 4,5 ind/ha (equivalente a un rango de 1,3 a $5 \mathrm{ind} / \mathrm{Km}$ de IARE). De este modo queda pendiente saber si fuera de éste rango el modelo lineal puede seguir siendo igualmente válido. La comparación de los valores correspondientes a un IARE lineal $( \pm 10 \%$ de intervalo de confianza) con los del IARE logarítmico evidencia que para DRs de alrededor de 4,5 ind/ha (equivalente a un IARE de 5 ind $/ \mathrm{Km}$ ), el IARE logarítmico cae fuera del intervalo de confianza correspondiente al IARE lineal y por tanto éste sería el límite por encima del cual el modelo lineal podría entrar en problemas de sobreestimación de la DR. Así, se podría concluir que si en una evaluación se encuentran IAREs que superan los 5 ind $/ \mathrm{Km}$ lo más recomendable sería analizar en detalle el tipo de relación existente entre DR e IARE ya que es probable que la existencia de una relación logarítmica cause problemas de sobreestimación de DR.

En la Figura 4 se muestra el diagrama de dispersión para la evaluación extensiva de FORCO habiéndose señalado las líneas de regresión correspondientes a los modelos lineal y potencial. Ambos modelos se muestran muy similares hasta una DR de alrededor de 3,5 ind/ha punto éste en el que el modelo lineal estaría sobreestimando a la DR con relación al modelo potencial.

De otro lado, conviene recordar que ya se señaló que el modelo lineal es un descriptor apropiado de la relación DR - IARE hasta una densidad de 4,5 ind/ha. Por tanto, sólo queda por verificar, para densidades superiores a la señalada, la posible existencia de una relación potencial. Esto se describe en la Figura 5, en la que es posible ver que para densidades superiores a 4,8 ind/ha (equivalente a IAREs del orden de 5 ind $/ \mathrm{Km}$ ) el IARE potencial se ubica fuera del intervalo de confianza del IARE lineal. Este sería el punto a partir del cual se puede caer en problemas de sobreestimación por la existencia de una relación potencial entre DR e IARE.

En el caso de MILO, además del modelo lineal $(\mathrm{r}=$ $0,6129, \mathrm{p}<0,05)$ los modelos potencial $(\mathrm{r}=0,6783$, $\mathrm{p}<0,01)$ y exponencial $(\mathrm{r}=0,6447, \mathrm{p}<0,01)$ alcanzan significación estadística y son candidatos a explicar la relación DR - IARE. Anteriormente, se ha explicado por qué resulta razonable escoger el modelo lineal a pesar de tener el valor de $\mathbf{r}$ más bajo pero con significación estadística.

El modelo lineal sugería que esta relación era válida para densidades reales entre 0 y $1,3 \mathrm{ind} / \mathrm{ha}$ (equivalentes a IAREs de entre 0 y 4 ind $/ \mathrm{Km}$ ). Si la relación no fuese lineal sino más bien potencial (Figura 6) DR, a partir de la cual los resultados de ambos modelos difieren, corresponde a 1,8 ind/ha (equivalente a un IARE de 3,5 ind/ $\mathrm{Km}$ ). Por encima de este límite el modelo lineal entraría en problemas de sobreestimación de la densidad. No obstante, el mensaje que se desprende de una posible relación exponencial (Figura 7) es exactamente el opuesto. En efecto, a partir de una densidad real de alrededor de $1,8 \mathrm{ind} / \mathrm{ha}$ (similar al límite sugerido por el modelo potencial) existe una diferencia entre el modelo lineal y exponencial que conduce a errores de estimación pero que a diferencia del modelo potencial, en este caso corresponden a errores de subestimación.

Por tanto, la densidad de 1,8 ind /ha equivalente a un IARE de 3,5 ind $/ \mathrm{Km}$, marca un límite por encima del cual se podría incurrir tanto en errores de sobreestimación como de subestimación. La recomendación que de esto se deriva es que si en una evaluación se encuentran IAREs superiores al límite mencionado será necesario realizar un ejercicio de calibración que permita identificar el tipo de relación entre DR e IARE evitando así errores de estimación.

En la evaluación intensiva, en el caso de FORCO el mejor modelo no lineal alternativo es el logarítmico $(\mathrm{r}=0,7048, \mathrm{p}<0,01)$.

De acuerdo al diagrama de dispersión las diferencias con el modelo lineal comienzan a partir de una DR de $60 \mathrm{ind} / \mathrm{ha}$. De otro lado, si se usa el criterio de comparar lo predicho por el modelo logarítmico con el modelo lineal $(+10 \%$ de intervalo de confianza) como se hace en la Figura 8, este punto se incrementa hasta un DR de $75 \mathrm{ind} /$ ha (equivalente a un IARE de $100 \mathrm{ind} / \mathrm{Km}$ ). Adicionalmente, conviene tener presente que el intervalo de validez del modelo lineal (cuyo $\mathrm{r}$ es de $0,7215, \mathrm{p}<0,01$ ) va de 0 a 100 ind/ha de DR. Esto equivale a un rango de IARE de 0 a más de $120 \mathrm{ind} / \mathrm{Km}$. Por tanto, si se comparan los tres criterios resulta que podemos estar razonablemente confiados en la validez del modelo lineal hasta IAREs del orden de $120 \mathrm{ind} / \mathrm{km}$; por encima de este límite sería necesario un proceso de calibración de la relación DR / IARE para descartar la existencia de relaciones logarítmicas.

Con relación a COCRU además del modelo lineal $(r=0,6113, p<0,05)$, el modelo exponencial es el modelo no lineal que alcanza más alta significación estadística en la descripción de la relación DR - IARE $(r=0,6288, p<0,05)$ por lo que es este modelo el que se usará para comparar con el modelo lineal. De acuerdo al diagrama de dispersión la relación lineal tiene validez para un intervalo de densidades que va 
desde $0 \mathrm{ind} / \mathrm{ha}$ hasta $470 \mathrm{ind} / \mathrm{ha}$, lo que equivale a IAREs entre 0 y $250 \mathrm{ind} / \mathrm{Km}$.

Si se toma en cuenta el criterio de la comparación con el modelo lineal $( \pm 10 \%)$, el modelo exponencial comienza a diferir significativamente del modelo lineal a partir de un DR de $450 \mathrm{ind} / \mathrm{ha}$. Por tanto, si tomamos en cuenta ambos criterios será posible afirmar que sólo a partir de $470 \mathrm{ind} /$ ha (equivalente a $250 \mathrm{ind} / \mathrm{Km}$ ) existe la posibilidad de que surjan problemas de subestimación. Entonces, es a partir de esta densidad que serán necesarios estudios de calibración que garanticen que la linealidad se mantiene.

En el espacio en el que se ha desarrollado el presente estudio resulta frecuente sentir los efectos del Evento El Niño (Torres y La Torre, 1994) los mismos que generan fuertes modificaciones en las densidades y distribuciones de las poblaciones de psitácidos en particular y de aves en general (Sánchez et al., 1998). La presencia de un ENSO puede desencadenar explosiones poblacionales (hot spots) que se traducen en densidades muy altas aunque sea de manera local.

De otro lado, la impredecibilidad en la oferta de agua asociada al ENSO puede obligar a que ciertas poblaciones se refugien en algunos lugares de hábitats favorables, cosa que también tiene como efecto un incremento local de la densidad poblacional. En ambas situaciones estamos frente a densidades altas y por tanto podríamos caer en los problemas de sobre y subestimación derivados de la existencia de relaciones no lineales entre DR e IARE. Esto plantea la necesidad de contar con estudios similares al presente justamente en los momentos en los que como consecuencia de un ENSO las densidades poblacionales se ven fuertemente afectadas. En todo caso, y sobre la base de la información recogida en el presente estudio se ha elaborado la Tabla 6 en la que para las tres especies evaluadas y para las dos modalidades de evaluación se han indicado los rangos de densidad en los cuales funciona el modelo lineal. Al mismo tiempo, se han indicado los modelos no lineales que con más probabilidad aparecerían por encima del rango anteriormente señalado y el tipo de problema (sobreestimación o subestimación) en el que se estaría incurriendo de usar un modelo lineal.

Se espera así contar con una guía que indique sobre la base de la información disponible - cuáles son los valores límite por encima de los cuales se podrían registrar no linealidades.

\section{Conclusiones.}

- Los histogramas de distribución de individuos, con relación a la línea de transecto, permitieron verificar el supuesto del método de Fourier de que las probabilidades de observación de un individuo son máximas sobre la línea del transecto disminuyendo conforme la distancia se incrementa.
- En general, para condiciones de densidades bajas la relación DR - IARE se ha mostrado lineal, por lo que la Abundancia Relativa es un buen estimador de la Densidad Real; para densidades altas surge la posibilidad de que modelos no lineales describan mejor la mencionada relación, generando así problemas de sobre y subestimación. Esto obliga a que por encima de estos límites, antes de usar IARE como indicador de DR, sea necesario comprobar experimentalmente la linealidad de la relación DR - IARE.

- En la evaluación extensiva de FORCO, se ha encontrado que los modelos no lineales logarítmico y potencial describen la relación IARE-DR de mejor manera que un modelo lineal. Ambos modelos a partir de IAREs mayores a 5 ind $/ \mathrm{km}$ (equivalentes a una DR de 4,5 ind $/ \mathrm{ha}$ ) podrían generar problemas de sobreestimación de DR.

- En la evaluación extensiva de MILO, los análisis sugieren que una densidad de 1,8 ind/ha (equivalente a un IARE de 3,5 ind/Km) marca el límite por encima del cual dos modelos no lineales (potencial y exponencial) podrían describir de mejor manera la relación IARE-DR. Si el modelo fuese potencial, el uso de un modelo lineal implicaría una sobrestimación de la densidad, en tanto que si el modelo fuese exponencial, se caería en una subestimación.

- La evaluación intensiva de FORCO sugiere que para densidades inferiores a $100 \mathrm{ind} / \mathrm{ha}$ (equivalentes a $120 \mathrm{ind} / \mathrm{Km}$ ) la relación DRIARE se mantiene lineal. Para densidades mayores, el modelo lineal podría dar paso a un modelo logarítmico y por consiguiente a problemas de sobreestimación de la densidad.

- Con relación a la evaluación intensiva de COCRU la densidad de $470 \mathrm{ind} / \mathrm{ha}$ (equivalente a $250 \mathrm{ind} / \mathrm{Km}$ ) marca el punto por encima del cual el modelo lineal podría ser reemplazado por un modelo exponencial generando problemas de subestimación de la densidad real.

\section{Referencias bibliográficas.}

Burnham K., Anderson D. \& Laake J. 1980. Estimation of density from line transect sampling of biological populations. Wildlife Monograph No. 72.

Caughley G. 1977. Analysis of vertebrate populations. John Wiley \& Sons. New Dehli.

Daniel, C. \& Wood F.. 1980. Fitting equations to data. John \& Sons. New York.

Falero M. y Sánchez E.. 1989-1990. El comercio de exportación de aves silvestres de la costa y sierra del Perú en el periodo 1982-1986. Zonas Aridas. (6): $109-134$.

Krebs Ch. 1989. Ecological Methodology. University of British Columbia. Harper Collins Publishers. 
McArdle B.H., Gaston K. \& Lawton J. 1990. Variations in the size of animal populations: patterns, problems and artifacts. Journal of Animal of Ecology. 59: 439 - 454.

Murdoch, W. 1994. Population regulation in theory and practice. Ecology. 75: $271-287$.

Norton-Griffiths M. 1978. Counting animals. Handbook $N^{\mathrm{o}}$ 1. Serengueti Ecological Monitoring Programme. Nairobi, Kenya.

Pimm S. 1991. The balance of nature? Ecological Issues in the conservation of species and communities. The University of Chicago Press. Chicago.

Pimm S. \& Redfearn, A. 1988. The variability of population densities. Nature. 334: $613-614$.

Quinteros Z. 1992. Determinación de los patrones de uso temporal y espacial de los cultivos de maíz por las aves granívoras en el campus de la UNALM. Tesis-Bióloga, Lima-Perú.

Rosales M. 1999. Análisis y alternativas de control de la comercialización de Psittacidos del Noroeste del Perú. Tesis $M g$. Sc. UNALM. Lima.

Sánchez E., Quinteros Z. y Falero M. 1998. Avances en la evaluación y manejo poblacional de Psitácidos (loros y pericos) en la Región Grau. Pp: 381 - 395 en: Bosques Secos y Desertificación. Memorias del Seminario Internacional. Proyecto Algarrobo INRENA. Lambayeque.
Sánchez E. y Quinteros Z. 1997. El desarrollo rural sustentable en el Perú desde la teoría ecológica. : 527 - 550 en: Perú el Problema Agrario en Debate. SEPIA VI. E. Gonzales de Olarte, B. Revesz y M. Tapia (Editores). Lima.

Sánchez E., Quinteros Z. y Sarabia J. 1992 a. Proyecto: Desarrollo de metodologías para la evaluación y manejo poblacional de psitácidos (loros y pericos) en la Región Grau. Laboratorio de Ecología Animal - UNALM, CEPESER, Direcciones Regionales Forestales y de Fauna de Piura y Tumbes. Lima.

Sinclair A. 1981. Enviromental carrying capacity and the evidence for overabundance. pp: 247-257. In : Problems in management of Locally Abundant Wild Animals. P.A. Jewell and S. Holt (Publisher). Academis Press, New York. NY.

Sinclair A.R.E. \& Peach R.P. 1996. Density dependence, stochasticity, compensation and predator regulation. Oikos. 75: $164-173$

Tellería J.L. 1986. Manual para el censo de los vertebrados terrestres. Editorial Raíces. Madrid.

Torres J. y La Torre, M 1994. Sinopsis del impacto El Niño en ecosistemas terrestres naturales $y$ artificiales. En: Seminario Taller Impacto Biológico de los Eventos El Niño en el Perú: Un reto para la ciencia y la tecnología. Mollendo, Arequipa.

Steel R. y Torrie J. 1985. Bioestadística: Principios y procedimientos. Mc Graw - Hill. Bogotá.

Tabla 1 Densidad Real e Indice de Abundancia Relativa. Evaluación Extensiva.

\begin{tabular}{|c|c|c|c|c|c|}
\hline \multicolumn{3}{|c|}{ FORCO } & \multicolumn{3}{|l|}{ MILO } \\
\hline Tramo & $\begin{array}{c}\text { DR } \\
\text { (ind/h) }\end{array}$ & $\begin{array}{c}\text { IARE } \\
\text { (ind/km) }\end{array}$ & Tramo & $\begin{array}{c}\text { DR } \\
\text { (ind/h) }\end{array}$ & $\begin{array}{c}\text { IARE } \\
\text { (ind/km) }\end{array}$ \\
\hline Fernández - Chicama & 4,3424 & 4,9167 & Barrancos -Qda.Fernández & 0,9753 & 1,3684 \\
\hline Encuentros - Bejucal & 0,2216 & 2,5000 & Bendito - Pizarro & 0,9613 & 3,3300 \\
\hline Jaguay Negro - Corral de Vaca & 0,6025 & 1,4706 & Casa Blanqueada - Rica Playa & 0,3095 & 1,3333 \\
\hline La Peñita (Burgos) - LaCancha & 0,1814 & 1,3529 & Encuentros - Bejucal & 0,3544 & 2,7000 \\
\hline Lancones - Encuentros & 0,1943 & 0,6458 & Fernández - Chicama & 0,5112 & 1,0588 \\
\hline Pampas de Hosp. - El Caucho & 0,3699 & 0,8500 & Jaguay Negro - Corral de Vaca & 1,3538 & 2,4706 \\
\hline Salados - La Peñita & 0,7777 & 1,4375 & Lancones.Poechos. Querecotillo & 0,2086 & 1,0323 \\
\hline Sauce Grande - El Angolo & 1,0930 & 4,2308 & Máncora - Barrancos & 1,1250 & 2,0000 \\
\hline & & & Pampas de Hospital - El Caucho & 1,0165 & 2,1000 \\
\hline & & & Papayal - Matapalo & 0,2036 & 0,9444 \\
\hline & & & San Jacinto - Casa Blanqueada & 1,1844 & 3,4545 \\
\hline & & & Sullana - Jaguay Negro & 0,8856 & 2,6250 \\
\hline & & & Zarumilla-Papayal & 0,4556 & 2,1667 \\
\hline & & & Zarumilla - Bendito & 0,4000 & 1,4167 \\
\hline
\end{tabular}


Tabla 2 Densidad Real e Indice de Abundancia Relativa.Evaluación Intensiva

\begin{tabular}{|c|c|c|c|c|c|}
\hline \multicolumn{3}{|c|}{ FORCO } & \multicolumn{3}{|c|}{ COCRU } \\
\hline TRAMOS & DR (ind/ha) & IARE (ind/Km) & TRAMOS & DR (ind/ha) & IARE (ind/Km) \\
\hline \multirow{6}{*}{$\begin{array}{l}\text { Sauce Grande } \\
\text { - El Angolo }\end{array}$} & 18,32 & 19,29 & \multirow{9}{*}{$\begin{array}{l}\text { Sauce Grande - El } \\
\text { Angolo }\end{array}$} & 173,17 & 92,50 \\
\hline & 10,11 & 31,82 & & 158,42 & 87,50 \\
\hline & 96,46 & 90,00 & & 110,15 & 265,00 \\
\hline & 16,43 & 36,00 & & 128,36 & 70,00 \\
\hline & 50,89 & 126,67 & & 171,82 & 112,50 \\
\hline & 12,92 & 42,22 & & 46,20 & 225,00 \\
\hline \multirow{3}{*}{$\begin{array}{l}\text { El Espino - } \\
\text { Barbacobas }\end{array}$} & 8,14 & 15,29 & & 386,37 & 285,00 \\
\hline & 36,70 & 72,86 & & 48,10 & 32,73 \\
\hline & 41,90 & 72,50 & & 60,60 & 41,11 \\
\hline \multirow{2}{*}{$\begin{array}{l}\text { Sauce Grande - } \\
\text { C.El Viento }\end{array}$} & 30,14 & 72,50 & \multirow{6}{*}{$\begin{array}{l}\text { El Espino - } \\
\text { Barbacobas }\end{array}$} & 198,16 & 93,33 \\
\hline & 5,64 & 11,05 & & 474,10 & 275,00 \\
\hline \multirow{4}{*}{$\begin{array}{l}\text { Sauce Grande } \\
\text { - La Tigra }\end{array}$} & 5,65 & 16,92 & & 118,07 & 75,00 \\
\hline & 4,95 & 45,71 & & 221,12 & 140,00 \\
\hline & 55,08 & 105,00 & & 288,87 & 275,00 \\
\hline & 4,28 & 73,33 & & 307,12 & 146,67 \\
\hline
\end{tabular}

Tabla 3 Estimación de densidad en transectos con series de Fourier. Tramo : Sauce Grande - El Angolo

\begin{tabular}{|c|c|c|c|c|c|c|c|}
\hline & & $K=1$ & $k=2$ & $k=3$ & $k=4$ & $k=5$ & $k=6$ \\
\hline$I$ & $x i$ & $\cos \left(k^{*} \mathrm{pi}^{*} \mathrm{xi} / \mathrm{w}\right)$ & $\operatorname{Cos}\left(k^{*} \mathrm{pi}^{*} \mathrm{xi} / \mathrm{w}\right)$ & $\cos \left(k^{*} \mathbf{p i}^{*} \mathbf{x i} / \mathbf{w}\right)$ & $\operatorname{Cos}\left(k^{*} \mathrm{pi}^{*} \mathrm{xi} / \mathrm{w}\right)$ & $\cos \left(k^{*} \mathbf{p i}^{*} \mathbf{x i} / \mathbf{w}\right)$ & $\cos \left(k * \mathrm{pi}^{*} \mathrm{xi} / \mathrm{w}\right)$ \\
\hline 1 & 10 & 0,8090 & 0,3090 & $-0,3090$ & $-0,8090$ & $-1,0000$ & $-0,8090$ \\
\hline 2 & 5 & 0,9511 & 0,8090 & 0,5878 & 0,3090 & 0,0000 & $-0,3090$ \\
\hline$\cdots$ & & & & & & & \\
\hline 54 & 35 & $-0,5878$ & $-0,3090$ & 0,9511 & $-0,8090$ & 0,0000 & 0,8090 \\
\hline 55 & 2 & 0,9921 & 0,9686 & 0,9298 & 0,8763 & 0,8090 & 0,7290 \\
\hline \multicolumn{2}{|c|}{ TOTAL } & 36,7440 & 23,5378 & 10,6176 & $-7,2203$ & $-7,4667$ & $-12,6361$ \\
\hline$\hat{A} k$ & & 0,026723 & 0,017118 & 0,007722 & $-0,005251$ & $-0,005430$ & $-0,009190$ \\
\hline \multicolumn{8}{|c|}{ sr $\quad=0,0038$ "stoping rule"(regla de detención; fija el número de términos de la serie a emplear) } \\
\hline \multicolumn{8}{|c|}{${ }^{\wedge} \mathrm{f}(0)=0,0517$ función de detección. } \\
\hline
\end{tabular}

Tabla 4 Coeficientes de determinación y correlación para diferentes modelos de relación DR-IARE. FORCO y MILO. Evaluación Extensiva.

\begin{tabular}{|l|c|c|c|c|c|c|}
\hline & \multicolumn{3}{|c|}{ FORCO } & \multicolumn{3}{c|}{ MILO } \\
\hline Modelos & Coef. Determinac. & Coef. Correl. & Significación & Coefic. Determinac. & Coef. Correl. & Significación \\
\hline Lineal & 0,6443 & 0,8027 & $*$ & 0,3756 & 0,6129 & $*$ \\
\hline Logarítmico & 0,6253 & 0,7908 & $*$ & 0,4019 & 0,6340 & $*$ \\
\hline Potencial & 0,525 & 0,7246 & $*$ & 0,4601 & 0,6783 & $* *$ \\
\hline Exponencial & 0,4712 & 0,6864 & Ns & 0,4157 & 0,6447 & $* *$ \\
\hline$*$ significativo al 5\%, $* *$ significativo al 1\%, ns no significativo.
\end{tabular}

Tabla 5 Coeficientes de determinación y correlación para diferentes modelos de relación DR - IARE. FORCO y COCRU. Evaluación Intensiva.

\begin{tabular}{|l|c|c|c|c|c|c|}
\hline & \multicolumn{3}{|c|}{ FORCO } & \multicolumn{3}{c|}{ COCRU } \\
\hline & $\begin{array}{c}\text { Coeficiente } \\
\text { Determinación }\end{array}$ & $\begin{array}{c}\text { Coeficiente } \\
\text { Correlación }\end{array}$ & $\begin{array}{c}\text { Signifi- } \\
\text { cación }\end{array}$ & $\begin{array}{c}\text { Coeficiente } \\
\text { Determinación }\end{array}$ & $\begin{array}{c}\text { Coeficiente } \\
\text { Correlación }\end{array}$ & $\begin{array}{c}\text { Signifi- } \\
\text { cación }\end{array}$ \\
\hline Modelo Lineal & 0,5206 & 0,7215 & $* *$ & 0,3737 & 0,6113 & $*$ \\
\hline Modelo Exponencial & 0,4395 & 0,6629 & $* *$ & 0,3954 & 0,6288 & $*$ \\
\hline Modelo Potencial & 0,4480 & 0,6693 & $* *$ & 0,3398 & 0,5829 & $*$ \\
\hline Modelo Logarítmico & 0,4968 & 0,7048 & $* *$ & 0,2473 & 0,4973 & $\mathrm{~ns}$ \\
\hline
\end{tabular}

* significativo al 5\%, ${ }^{*}$ significativo al $1 \%$, ns no significativo 
Diciembre 2002

Tabla 6 Límites de validez para la linealidad entre DR / IARE; modelos alternativos y problemas de estimación correspondientes.

\begin{tabular}{|l|c|c|c|c|}
\hline \multicolumn{1}{|c|}{ Especie } & \multicolumn{2}{|c|}{ Límites para la linealidad } & Modelo no lineal alternativo & Efecto sobre la estimación \\
\hline FORCO, Eval. Extensiva & $5 \mathrm{ind} / \mathrm{Km}$ & $4.5 \mathrm{ind} / \mathrm{ha}$ & Logarítmico & Sobreestimación \\
& $5 \mathrm{ind} / \mathrm{Km}$ & $4.5 \mathrm{ind} / \mathrm{ha}$ & Potencial & Sobrestimación \\
\hline MILO, Eval. Extensiva & $3.5 \mathrm{ind} / \mathrm{Km}$ & $1.8 \mathrm{ind} / \mathrm{ha}$ & Potencial & Subestimación \\
& $3.5 \mathrm{ind} / \mathrm{Km}$ & $1.8 \mathrm{ind} / \mathrm{ha}$ & Exponencial & Sobreestimación \\
\hline FORCO, Eval. Intensiva & $120 \mathrm{ind} / \mathrm{Km}$ & $100 \mathrm{ind} / \mathrm{ha}$ & Logarítmico & Subestimación \\
\hline COCRU, Eval.Intensiva & $250 \mathrm{ind} / \mathrm{Km}$ & $470 \mathrm{ind} / \mathrm{ha}$ & Exponencial & \multicolumn{3}{c}{}
\end{tabular}

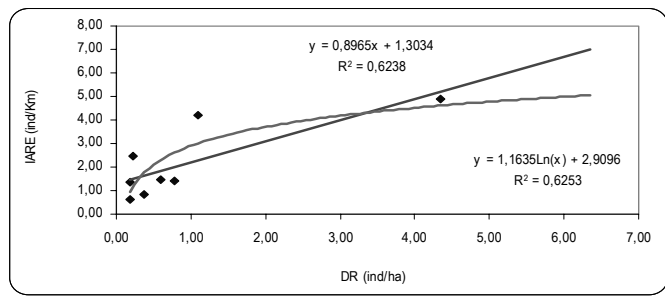

Figura 1 Relación DR-IARE. FORCO.

Eval.Extensiva.Modelos Lineal y Logarítmico.

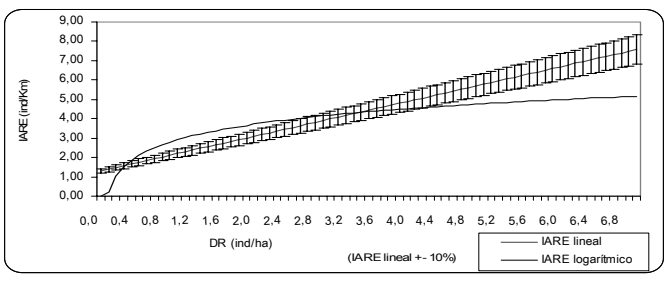

Figura 3 Estimación de DR con un modelo lineal y uno logarítmico. FORCO. Eval. Ext.

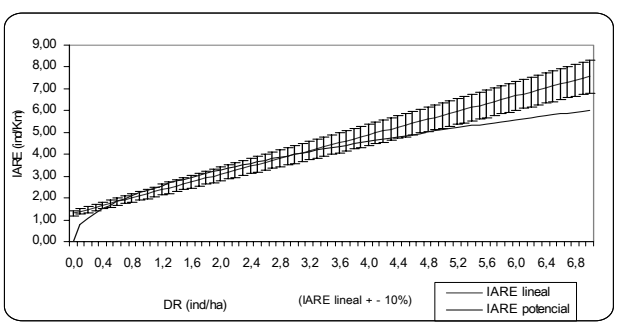

Figura 5 Estimación de DR con un modelo lineal y uno potencial. FORCO. Eval. Extensiva.

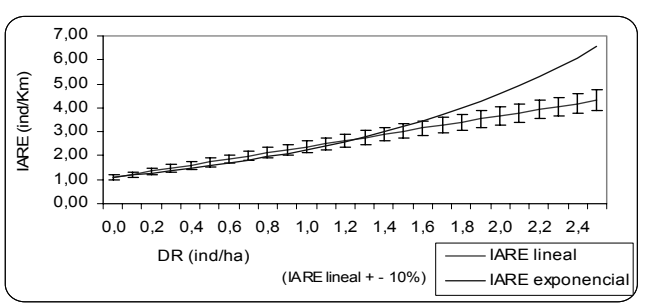

Figura 7 Estimación de DR con un modelo lineal y uno exponencial. MILO. Eval. Extensiva.

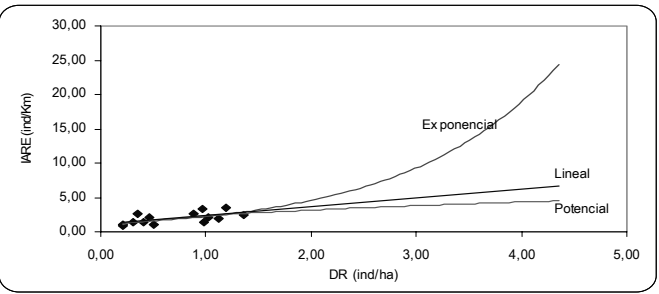

Figura 2 Relación DR / IARE para MILO. Eval. Extensiva. Modelos no lineales.

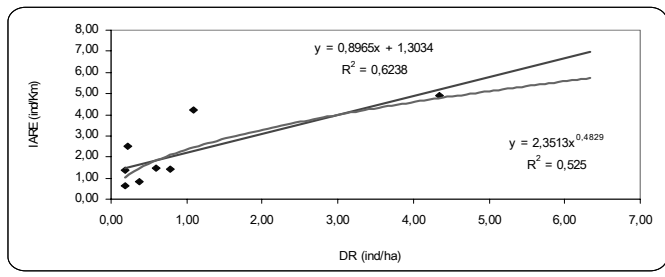

Figura 4 Relación DR-IARE. FORCO.

Eval.Extensiva. Modelos Lineal y Potencial.

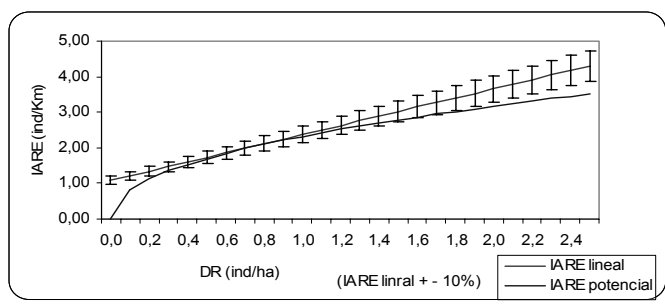

Figura 6 Estimación de DR con un modelo lineal y uno potencial. MILO. Eval. Extensiva.

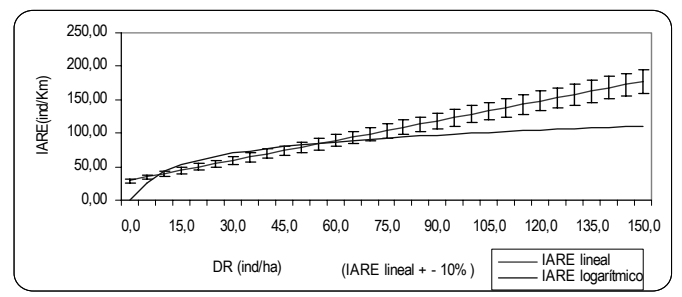

Figura 8 Estimación de DR con un modelo lineal y uno logarítmico. FORCO. Eval.Intensiva. 